\title{
Desarrollo de habilidades científicas en la formación inicial de profesores de ciencias y matemática ${ }^{1}$
}

David Reyes-González

Universidad Metropolitana de Ciencias de la Educación, Chile david.reyes@umce.cl
Yonnhatan García-Cartagena

Universidad Metropolitana de Ciencias de la Educación, Chile jonnhatan.garcia@umce.cl

\section{Resumen}

Estudio que indaga las habilidades científicas en un curso introductorio de robótica en la formación de profesores de física, química y matemática. La investigación se estructuró en dos fases: capacitación en aspectos propios de la robótica (electrónica y programación) mediante el uso de los kits Home-Bot ${ }^{\circledR}$ de Parallax ${ }^{\circledR}$ y el lenguaje de programación PBASIC; $y$ una fase de resolución de problemas prácticos de navegación con robots.

La recolección de información se realizó mediante una prueba escrita y una lista de cotejo, además de registros audiovisuales. El análisis se llevó a cabo utilizando una distribución de frecuencias de observación de la habilidad y sus medias estadisticas, así como las pruebas de comparación de medidas entre grupos. Al final se realizó una comparación entre datos cuantitativos y cualitativos extraídos mediante el análisis de contenido en los registros en video.

Entre los principales resultados se encuentran: que las habilidades testeadas se manifiestan sin diferencia significativa entre estudiantes de las carreras de pedagogía en física, pedagogía en química y pedagogía en matemática; hechos que destacan el potencial de la robótica para desarrollar habilidades de proceso cientifico en la formación inicial de profesores de ciencias y matemática.

\section{Palabras clave}

Actividad cientifica, formación del personal docente, robótica, desarrollo de habilidades. (Fuente: Tesauro de la Unesco).

Recepción: 2014-03-12 / Envío a pares: 2014-06-19 / Aceptación por pares: 2014-06-28 / Aprobación: 2014-07-17 DOI: 10.5294/edu.2014.17.2.4

Para citar este artículo / To reference this article / Para citar este artigo

Reyes-González, D. y García-Cartagena, Y. (2014). Desarrollo de habilidades científicas en la formación inicial de profesores de ciencias y matemática. Educ. Educ. 17 (2), 271-285. Doi. 10.5294/edu.2014.17.2.4

$1 \quad$ Los autores agradecen a la Dirección de Investigación de la Universidad Metropolitana de Ciencias de la Educación por el financiamiento otorgado mediante el proyecto FIBE 14-12 del que se desprenden los resultados reportados. 


\title{
Development of Scientific Skills in Initial Training for Mathematics and Science Teachers
}

\begin{abstract}
The study examined the scientific skills in an introductory robotics training course for physics, chemistry and mathematics teachers. The research was divided into two phases: training on the particular aspects of robotics (electronics and programming), using the Home-Bot ${ }^{\circledR}$ Paralla ${ }^{\circledR}$ and PBASIC programming language kits; and a practical problem-solving phase involving navigation with robots.

The data were collected by means of a written test and a checklist, in addition to audio recordings. This information was analyzed using a frequency distribution for the skill and its statistical measurements, combined with tests comparing the measurements among groups. Last of all, a comparison between the quantitative and qualitative data extracted in the study was done by analyzing the contents of the video recordings.

With regard to the main results, the skills being tested were manifest with no significant differences among those who are studying for teaching careers in physics, chemistry pedagogy and mathematics. This highlights the potential of robotics to develop science process skills in initial training for science and mathematics teachers.
\end{abstract}

\section{Key words}

Scientific activities, educational personnel training, robotics, Skills development. (Source: Unesco Thesaurus). 


\section{Desenvolvimento de habilidades científicas na formação inicial de professores de ciências e matemática}

\section{Resumo}

Estudo que questiona as habilidades cientificas num curso introdutório de robótica na formação de professores de física, química e matemática. A pesquisa foi estruturada em duas fases: capacitação em aspectos próprios da robótica (eletrônica e programação) mediante o uso dos kits Home-Bot ${ }^{\circledR}$ de Parallax ${ }^{\circledR}$ e a linguagem de programação PBASIC; e uma fase de resolução de problemas práticos de navegação com robôs.

A coleta de informação foi realizada mediante uma prova escrita e uma lista de cotejo, além de registros audiovisuais. A análise foi realizada utilizando uma distribuição de frequências de observação da habilidade e suas médias estatísticas, bem como as provas de comparação de medidas entre grupos. Ao final, foi feita uma comparação entre dados quantitativos e qualitativos extraidos mediante a análise de conteúdo nos registros em vídeo.

Entre os principais resultados, encontram-se que: as habilidades testadas se manifestam sem diferença significativa entre estudantes dos cursos acadêmicos de pedagogia em física, em química e em matemática; fatos que destacam o potencial da robótica para desenvolver habilidades de processo cientifico na formação inicial de professores de ciências e matemática.

\section{Palavras-chave}

Atividades científicas, formação de docentes, robótica, desenvolvimento de competências. (Fonte: Tesauro da Unesco). 
ISSN 0123-1294 | Educ.Educ. Vol. 17. No. 2 | Mayo-Agosto de 2014 | pp. 271-285.

Universidad de La Sabana | Facultad de Educación

\section{La robótica en los espacios formativos}

La robótica educativa (RE) se puede definir como un área de la pedagogía que incorpora a la robótica y la automatización de procesos como un elemento mediador para el logro de aprendizajes en los procesos formativos (García y Reyes, 2012). En este punto cabe destacar que la RE ha sido empleada de forma transversal para desarrollar un conjunto de habilidades que se corresponden con aquellas requeridas para el siglo XXI, entre otras, el razonamiento crítico (Sendag et al., 2009) y la resolución de problemas (Wagner, 1998).

La robótica en sí misma es multidisciplinaria. Un robot se constituye como un sistema con diversas partes, motores, sensores y un programa que lo gobierna, entre otros. Cada una de estas partes depende de diferentes campos del conocimiento tales como la ingeniería, la electrónica y la informática. Este carácter interdisciplinar significa que cuando los estudiantes aprenden a diseñar robots, inevitablemente aprenden sobre las muchas otras disciplinas que confluyen en la robótica (Papert, 1980; Rogers y Portsmore, 2004). De la misma forma, la construcción de robots por los estudiantes les enseña que todas las partes de un sistema complejo interactúan y dependen unas de otras (Beer et al., 1999; Sullivan, 2008). Esta última es una habilidad requerida para cualquier persona que necesite comprender sistemas complejos, sea en biología, ingeniería, sociología u otras disciplinas.

Con todo, según la NRC (1996), el diseño tecnológico es paralelo a la indagación en ciencias. De esta forma los estudiantes, a través de la resolución de problemas en robótica y el diseño de sistemas robóticos, se ven involucrados en actividades equivalentes a las de indagación científica. Por otra parte, según Haapasalo y Samuels (2011), la robótica proporciona un ambiente constructivista para el aprendizaje de conceptos matemáticos.

Otras investigaciones que involucran a la robótica en el contexto educacional han mostrado que existe un efecto positivo en la motivación en el aula (Barker y Ansorge, 2007; Carbonaro, Rex y Chambers, 2004; Gura, 2007; Nourbakhsh et al., 2005), como también su efectividad potencial para impactar positivamente en el aprendizaje (Fagin y Merkle, 2003). En este contexto, múltiples autores sostienen que el ambiente de aprendizaje generado permite establecer que la robótica es intelectualmente rica (Barnes, 2002; Beer et al., 1999; Chambers y Carbonaro, 2003; Flake, 1990; Flowers y Gossett, 2002; García y McNeill, 2002; Klassner, 2002; Kumar, 2004; Nourbakhsh, 2000; Miglino et al., 1999; Resnick y Ocko, 1991; Shimbakuro, 1989; Wagner, 1998; Weinberg et al., 2005; Ringwood et al., 2005; Sargent et al., 1996).

Siguiendo esta línea, existen estudios que muestran cómo la robótica promueve el interés por las carreras de matemáticas y ciencias (Barnes, 2002; Robinson, 2005; Rogers y Portsmore, 2004). La robótica también promueve el aprendizaje de los principios científicos y matemáticos a través de la experimentación (Rogers y Portsmore, 2004), invita a la resolución de problemas (Barnes, 2002; Mauch, 2001; Nourbakhsh et al., 2005; Robinson, 2005; Rogers y Portsmore, 2004) y promueve el aprendizaje cooperativo (Beer et al., 1999; Nourbakhsh et al., 2005). No obstante los beneficios positivos tanto en la instrucción como en la motivación, a partir de estos estudios también se observa que existen escasos reportes sobre aplicaciones de la robótica en la formación inicial docente de profesores de ciencias y su potencial para el desarrollo y fortalecimiento de habilidades científicas. En síntesis, este es el núcleo central del problema que pretendemos abordar a partir de este estudio.

\section{Habilidad científica y robótica}

En la literatura se destaca que la habilidad es un concepto en el cual se vinculan aspectos psicológicos y pedagógicos indisolublemente unidos. Desde el punto de vista psicológico hablamos de las acciones y operaciones, y desde una concepción pedagógica, el cómo dirigir el proceso de asimilación de 
esas acciones y operaciones. La acción es una unidad de análisis que se da solo cuando el individuo actúa y se puede descomponer en varias operaciones con determinada lógica y secuencia. Cada habilidad posee operaciones cuya integración permite el dominio de un modo de actuación por los estudiantes (Cañedo Iglesias, 2008).

Por las características multidisciplinarias y sus efectos sobre la motivación de los estudiantes, la robótica proporciona múltiples ventajas al momento de desarrollar contenidos y habilidades particulares. Respecto de los contenidos, la literatura reporta estudios asociados al desarrollo de la cinemática y la dinámica, circuitos eléctricos, sistemas mecánicos, análisis y construcción de gráficos, sistemas complejos, algoritmos y resolución de problemas, diseño y arquitectura, entre otros. Respecto de las habilidades científicas, según Sendag y Odabasi (2009), las habilidades de orden superior como resolución de problemas, pensamiento creativo y pensamiento crítico son parte importante de las habilidades que pueden movilizarse siempre que se genere un contexto adecuado mediante la robótica educativa. Así mismo, según Sullivan (2008), la resolución de problemas en robótica, como recurso didáctico, involucra cuatro de seis habilidades características de la alfabetización científica: computación, estimación, manipulación y observación.

Cuando se trabaja con robots, las actividades principales incluyen la manipulación de herramientas, el uso de computadores, los sensores, la estimación de variables, entre muchas más. Cuando los estudiantes tienen que solucionar problemas de navegación con un sistema robótico deben hacer medidas y cálculos numéricos basados en las configuraciones de los sensores y estimar variables para poder escribir el programa que evalúa dichas variables y gobierna el comportamiento del sistema. Con estos resultados en mano, los estudiantes observan las respuestas del sistema y realizan los ajustes necesarios obteniendo retroalimentación de forma inmediata en el proceso formativo.
Finalmente, si se acepta que el estudiar ciencias naturales implica un proceso de razonamiento bajo cierta lógica, que incluye un conjunto de habilidades como la formulación de hipótesis, inferir, predecir y obtener conclusiones (tabla 1), entonces es posible que a través de la robótica en la formación inicial docente puedan potenciarse y fortalecerse aquellas habilidades relacionadas con el proceso de hacer ciencia.

En el plano local, un profesor de ciencias debe desarrollar un sus estudiantes un conjunto de habilidades definidas en las bases curriculares vigentes (Mineduc, 2012). De acuerdo con estas, el estudio de sus disciplinas implica un proceso de razonamiento lógico que incluye un conjunto de habilidades (última columna tabla 1) entre las que se encuentran la formulación de hipótesis, inferir, predecir y obtener conclusiones, entre otras.

Un punto importante por considerar son los aspectos de género relacionados con el hacer ciencia. No obstante, en la literatura reciente se indica que estas diferencias están relacionadas con aspectos culturales (Else-Quest et al., 2010). Los resultados de la prueba PISA 2012 en ciencias confirman esta tendencia, y que para el caso chileno las diferencias de género no son significativas (OCDE, 2013).

La adquisición progresiva de las habilidades científicas está enfocada hacia la alfabetización científica que corresponde a la capacidad de aplicar en su ambiente cotidiano los conocimientos y las habilidades que les permitan tomar decisiones informadas y que afectan su entorno familiar y su comunidad (García y Reyes, 2012).

Del análisis de las habilidades de proceso científico que se reportan en la literatura es factible suponer que la ciencia, en su proceso, se compone de fases en las que: 1) se identifica un problema de investigación o fenómeno por investigar para el cual se 2) generan formas de abordarlo y analizarlo y cuyos 3) resultados son socializados; estos resultados eventualmente pueden ser utilizados para definir 
Tabla 1. Sintesis de habilidades de proceso científico reportadas en la literatura reciente

\begin{tabular}{|c|c|c|c|c|c|}
\hline Abruscato (2004) & $\begin{array}{l}\text { Friedl y Koontz } \\
\text { (2005) }\end{array}$ & $\begin{array}{l}\text { Chiappetta y } \\
\text { Koballa (2006) }\end{array}$ & $\begin{array}{l}\text { Martin et al. } \\
\quad(2009)\end{array}$ & $\begin{array}{l}\text { Kovalik y Olsen } \\
(2010)\end{array}$ & Mineduc (2012) \\
\hline Observar & Observar & Observar & Observar & Observar & Observar \\
\hline Clasificar & Clasificar & Clasificar & Clasificar & Comunicar & Clasificar \\
\hline Predecir & Inferir & Usar números & Predecir & Comparar & Comunicar \\
\hline Usar números & Comunicar & Medir & Usar números & Organizar & Medir \\
\hline Medir & Medir & Inferir & Medir & categorizar) & Usar modelos \\
\hline Inferir & Experimentar & Usar relaciones & Interpretar datos & Relacionar & Experimentar \\
\hline $\begin{array}{l}\text { Usar relaciones } \\
\text { espacio/tiempo }\end{array}$ & & Interpretar datos & $\begin{array}{l}\text { Controlar } \\
\text { variables }\end{array}$ & Inferir & Analizar \\
\hline Comunicar & & Controlar variables & Definir & Apl & Colmipald \\
\hline Interpretar datos & & Hipotetizar & & & \\
\hline Controlar variables & & Definir & & & Expioldi \\
\hline Hipotetizar & & operacionalmente & Formular modelos & & $\begin{array}{l}\text { Formular } \\
\text { preguntas }\end{array}$ \\
\hline Definir & & Experimentar & Interır & & Investigar \\
\hline operacion & & Formular modelos & Coms & & Planificar \\
\hline Experimentar & & & Preguntar & & Registrar \\
\hline & & & & & $\begin{array}{c}\text { Usar } \\
\text { instrumentos }\end{array}$ \\
\hline
\end{tabular}

nuevos proyectos de investigación. De este modo, hacer ciencia hoy en día se puede considerar como un proceso que reúne un conjunto de habilidades denominadas habilidades del proceso científico y que, en términos de habilidades accesibles mediante la observación, se plantea que a partir de ellas es posible describir cinco grandes categorías en el proceso científico. Estas categorías se pueden clasificar en tres permanentes o cuya transición es relativamente larga durante el proceso, a saber: observar, estudiar y comunicar, y dos categorías de transición, a saber: problematización y codificación (figura 1).

A continuación se describen las principales características de las categorías o fases en el proceso de cinco fases antes mencionado.
Figura 1. Habilidades de proceso científico

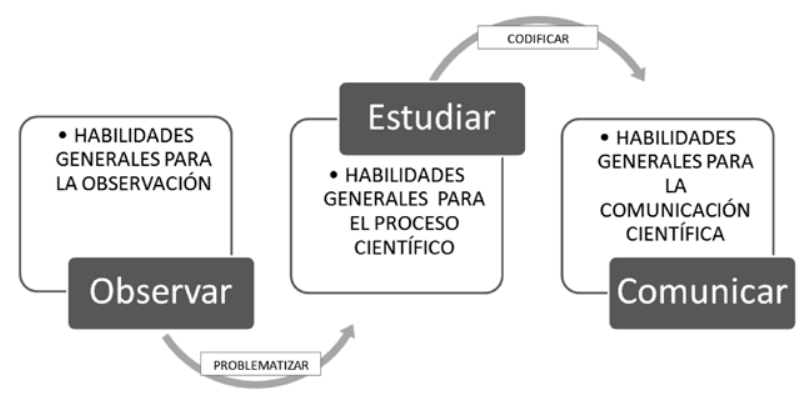

La habilidad para observar comprende la utilización de la totalidad de los sentidos en la distinción de patrones mediante el examen, la identificación, el reconocimiento, la comparación y la contrastación, entre otras, en el dominio intelectual y afectivo. 
Si los instrumentos se consideran como una extensión, ampliación, complementación u optimización de los sentidos, entonces el usar instrumentos corresponde a una forma particular de observación que se da en el dominio de lo intelectual (razonamiento de acuerdo con determinado estándar), psicomotriz (operación con instrumental) y afectivo (motivación o involucramiento con la actividad). Problematizar correspondería a la primera categoría de transición entre observar y estudiar. En esta parte del proceso los involucrados en la resolución de un problema deben delimitar e identificar claramente el problema central que se va a estudiar. En esta fase de transición se espera la formulación de hipótesis, predicciones basadas en la observación, el planteamiento de preguntas que permitan identificar el objeto de estudio y la delimitación del problema.

La habilidad de estudiar un problema desde la perspectiva científica está íntimamente relacionada con sus resultados, a saber: generar algún tipo de conocimiento nuevo o mejorado respecto de un fenómeno natural o técnico específico. Estas habilidades generales se dan en el ámbito intelectual (aprendizaje: cognición y metacognición), psicomotriz (manipulación u operación con instrumental), afectivo (actitud hacia la ciencia, motivación) y social (conocimiento colectivo, capital social, alfabetización científico-tecnológica).

Desde esta perspectiva, las habilidades de proceso científico constituyen el conjunto de habilidades básicas por desarrollar a fin de abordar un problema en el contexto científico-técnico actual. Este grupo de habilidades generales involucran la inferencia, la exploración, la experimentación (falsación de hipótesis, control de variables, etc.), el registro de observaciones o datos, la clasificación, la predicción, el uso de modelos explicativos-predictivos, el análisis, la síntesis, la evaluación de respuestas o modelos alternativos. También involucra el trabajo individual o colectivo para la investigación o el desarrollo en los ámbitos científico-técnico.
Codificar corresponde a la segunda categoría de transición entre estudiar y comunicar. Toda comunicación debe realizarse de acuerdo a un estándar basado en códigos lingüísticos adecuados al contexto en que se sustenta la comunicación. Para el caso de la comunicación científica el estándar supone la utilización de tablas o gráficos, lenguaje numérico (uso de números) o de relaciones entre un conjunto de entidades (variables), así como las herramientas infocomunicacionales para registrar $y$, posteriormente, comunicar los hallazgos relativos al proceso de estudiar un fenómeno científico-técnico particular.

Por último, entendido el acto de comunicar como una relación dada en el dominio social, para que este acto se manifieste se requiere la participación de por lo menos un ente comunicante que envía un mensaje a un ente receptor en un contexto específico, la habilidad de comunicar de acuerdo con las normas científicas implica conocer el lenguaje de las ciencias para generar un mensaje con contenido científico contextualizado, de forma tal que el emisor y receptor interactúen de acuerdo con el contenido de ese mensaje. De esta forma, no es factible observar el acto comunicativo sin los componentes antes mencionados, a saber: contexto, emisor, mensaje y receptor.

Así pues, se puede establecer que en el acto de comunicar científicamente es necesario por lo menos lo siguiente: la utilización de tablas o gráficos, la interpretación de datos, la utilización de números o relaciones numéricas, el uso de modelos explicativos, la utilización de definiciones operacionales, la formulación de preguntas ad-hoc al contexto.

De esta forma, y para este estudio, el conjunto de habilidades por estudiar se muestran en la tabla 2.

\section{Metodología}

Este es un estudio exploratorio que indaga acerca de las habilidades de proceso científico y su potencial para ser desarrolladas y fortalecidas en el 
ISSN 0123-1294 | Educ.Educ. Vol. 17. No. 2 | Mayo-Agosto de 2014 | pp. 271-285.

Universidad de La Sabana | Facultad de Educación

Tabla 2. Habilidades de proceso científico consideradas en este estudio para cada etapa del proceso

\begin{tabular}{|c|c|c|c|c|}
\hline Observar & Problematizar & Estudiar & Codificar & Comunicar \\
\hline Medir & Plantear objetivos & Predecir & Usar gráficos y tablas & Usar números \\
\hline Comparar & Formular preguntas & Inferir & Registrar & $\begin{array}{c}\text { Definir } \\
\text { operacionalmente }\end{array}$ \\
\hline \multirow[t]{5}{*}{ Explorar } & Planificar & Controlar variables & & $\begin{array}{c}\text { Formular preguntas } \\
\text { (diálogo con pares) }\end{array}$ \\
\hline & Plantear objetivos & Clasificar & & \\
\hline & $\begin{array}{c}\text { Delimitar el } \\
\text { problema }\end{array}$ & Usar modelos & & \\
\hline & & Explorar & & \\
\hline & & Analizar & & \\
\hline
\end{tabular}

contexto de un curso de introducción a la robótica implementado en la formación inicial de profesores de física, química y matemática en la Universidad Metropolitana de Ciencias de la Educación en Santiago de Chile. Para lograr este objetivo la investigación se estructuró en dos fases: 1) capacitación en aspectos propios de la robótica (electrónica y programación) mediante el uso de los kits Home-Bot ${ }^{\circledR}$ de Paralla ${ }^{\circledR}$ y el lenguaje de programación PBASIC,y posteriormente una fase de 2 ) resolución de problemas prácticos de navegación con robots.

La recolección de información se realizó mediante una prueba escrita aplicada al final del curso, una lista de cotejo para llevar registro de las habilidades evidenciadas - ambas diseñadas a propósito-, además de registros en video a fin de obtener información complementaria. Para el análisis de los datos se utilizaron los criterios de frecuencia de observación de las habilidades, correlación de Pearson para cada habilidad y el género de los participantes, así como para cada habilidad y la carrera de pedagogía que cursan los sujetos estudiados, y prueba $t$ de Student para probar las hipótesis planteadas, a saber: 1) no existe diferencia significativa entre las habilidades evidenciadas y el género de los sujetos, y 2) no hay diferencia significativa entre las habilidades evidenciadas según si los sujetos cursan la carrera de pedagogía en física o en química o en matemática. Finalmente, se contempló la comparación de datos cualitativos y cuantitativos como una forma general de evaluación de la consistencia interna de los resultados obtenidos.

\section{Procedimiento y muestra}

La población en estudio está representada por estudiantes de pedagogía en física, química, biología y matemática de la Universidad Metropolitana de Ciencias de la Educación ${ }^{2}$, principal universidad formadora de profesores en Chile. Para la selección de la muestra se procedió según si los sujetos: 1) estaban o no en el mismo nivel de estudios o semestre de sus carreras, 2) que las cuatro carreras de pedagogía en estudio tuvieran un número similar de representantes, y finalmente, 3) según el interés de participar y la disponibilidad de horarios por parte de los estudiantes.

Finalmente, la muestra quedó constituida por un grupo de 19 estudiantes: 6 de pedagogía en física (PF), 6 de pedagogía en química (PO) y 7 de pedagogía en matemática (PM) que se encontraban en el octavo semestre de sus respetivas carreras. De estos, 11 fueron mujeres. No se inscribió ningún estudiante de pedagogía en biología. Un estudiante no finalizó el curso y no participó de las evaluaciones.

2 www.umce.cl. 
El procedimiento empleado en este estudio involucra dos fases:

1. Fase de inducción o capacitación: en esta etapa se inicia la capacitación en el uso de herramientas para el armado de circuitos electrónicos, construcción de sensores, software para la programación, y la implementación de rutinas de navegación autónoma de los robots. La capacitación se dividió en diez sesiones de tres horas semanales, en las que los estudiantes nivelan sus conocimientos en electrónica básica (circuitos y herramientas) y programación de microcontroladores. En el curso se conformaron diez grupos de dos estudiantes, cada uno con un kit robótico y una computadora portátil. En cada sesión participa un instructor que desarrolla los contenidos de la capacitación y registra habilidades a partir de la lista de cotejo y los desempeños especificados para cada habilidad.

2. Fase de resolución de problemas y recolección de datos para la investigación: aquí los estudiantes son sometidos a pruebas que implican la resolución de problemas de navegación que no les han sido presentados en la fase de inducción. Estos problemas han sido ideados de forma tal que representen un desafío al ingenio, y que puedan ser abordados con el conocimiento adquirido en la fase de nivelación, por lo que el problema-desafío representa una situación donde se ponen en juego las habilidades con tal de resolver el problema de forma total o parcial.

\section{Recogida de datos}

Se realizó un registro audiovisual por medio de grabación de dos problemas prácticos o desafíos que los estudiantes tuvieron que resolver. El primer problema estaba asociado a la navegación del robot utilizando sensores de tacto para salir de un laberinto, y el segundo a la navegación del robot utilizando sensores de intensidad luminosa.
Adicionalmente se construyó una lista de cotejo con base en las habilidades de proceso científico reportadas por la literatura reciente (tabla 2), para generar los criterios de desempeño que se debían observar. Esta lista de cotejo permitió identificar qué habilidades se manifestaron cuando los sujetos en estudio se encontraban solucionado un problema de navegación. A su vez, este instrumento sirvió para identificar las habilidades presentadas en el registro de video.

También se aplicó un test de habilidades científicas con base en la teoría clásica de la medición y teoría de respuesta al item. Este instrumento solo consideró aquellas habilidades que podrían ser evaluadas mediante una prueba escrita sin grandes dificultades metodológicas; dichas habilidades representan un subconjunto de las presentadas en la lista de cotejo. A partir de este instrumento fue posible comparar las frecuencias de observación y sus correlaciones con el género y las carreras de los sujetos en estudio.

\section{Análisis y resultados}

Del conjunto de habilidades que se pretendía observar utilizando la lista de cotejo durante las actividades de resolución de problemas solo dos no pudieron ser observadas. Estas son: "Definir operacionalmente" e "Hipotetizar".

La afirmación de que no se observan las habilidades no implica que la habilidad no pueda ser movilizada por la resolución de problemas con robótica o que no se pueda desarrollar mediante esta actividad. Solamente indica que mediante la técnica de observación y registro audiovisual no es posible observar dicha habilidad.

Por ejemplo, la habilidad de proceso científico denominada hipotetizar, es decir, el planteamiento de explicaciones que son susceptibles de comprobar, puede ocurrir en el ámbito de lo cognitivo, como un proceso interno de los sujetos, y mediante la observación del registro audiovisual solo se tiene acce- 


\section{Tabla 3. Conjunto de habilidades por observar según la lista de cotejo y el resultado de la observación}

\begin{tabular}{|c|c|}
\hline Habilidades & Observación \\
\hline Medir & Se observa \\
\hline Explorar & Se observa \\
\hline Formular preguntas & Se observa \\
\hline Delimitar problema & Se observa \\
\hline Hipotetizar & No se observa \\
\hline Planificar & Se observa \\
\hline Controlar variables & Se observa \\
\hline Usar modelos & Se observa \\
\hline Clasificar & Se observa \\
\hline Usar gráficos y tablas & Se observa parcialmente \\
\hline Registrar & Se observa \\
\hline Usar números & Se observa \\
\hline Definir operacionalmente & No se observa \\
\hline
\end{tabular}

so al tramo externo observable de dicha habilidad, es decir, cuando la explicación es sometida a una prueba. En muchas ocasiones los sujetos probaban el programa introducido al robot para observar su comportamiento y realizar modificaciones o probar que funcionaba correctamente (debugging). No es posible comprobar si la acción de someter a prueba el robot es producto del planteamiento de una hipótesis o solo es por ensayo-error. También puede ocurrir el acto inverso, es decir, luego de observar la conducta del programa introducido al robot y percibir un comportamiento "extraño" o no planeado, los estudiantes podrían plantearse explicaciones susceptibles de comprobar. Sobre esta habilidad específica se estima que tendrían que diseñarse instrumentos de recogida de información que permitan hacer explícitas ciertas habilidades en los estudiantes, por ejemplo, entrevistas personales.

También se reportó como parcial la habilidad de "usar tablas y gráficos" debiso a que los estudiantes no tuvieron que realizar representaciones gráficas en el contexto de la resolución de problemas con robots. De esta habilidad solo se observó que los alumnos tabulaban ciertos datos, para el caso, la intensidad luminosa medida a partir de fotorresistores.

En cuanto a las habilidades evaluadas a partir de la prueba escrita cotejada según la carrera que cursan los sujetos, la habilidad "Aplicar" se encuentra en el $86 \%$ de los que cursan pedagogía en matemática (PM), el $75 \%$ de los sujetos que cursan pedagogía en física (PF), y en el $50 \%$ de los sujetos que cursan pedagogía en química (PO). La habilidad "Comparar" se evidencia en el $65 \%$ de los estudiantes de PM, en el $100 \%$ de los estudiantes de PF y en el $67 \%$ de los estudiantes de PO. La tabla 4 sintetiza los resultados para todas las habilidades testeadas.

La habilidad "Predecir" se observa con mayor frecuencia en estudiantes de PM y de PF, existiendo una diferencia significativa $(p=0,036)$ entre estudiantes de $\mathrm{PQ}$ y PM. Esta diferencia es más significativa $(p=0,016)$ cuando se comparan estudiantes de PQ y PF. Estas diferencias desaparecen cuando se eliminan los datos que están fuera de la desviación estándar. Por otra parte, la habilidad "Comparar" aparece con una diferencia significativa $(p=0,042)$ entre estudiante de PF en comparación con PM, pero esta no es significativa para PF comparada con estudiantes de PQ. Las restantes habilidades no presen$\tan$ diferencias significativas con $p=0,05$.

Por tanto, se podría establecer que una vez conocida la excepción se puede encontrar por regla que no existe diferencia significativa en la manifestación de las habilidades testeadas entre estudiantes de las carreras representadas en la muestra.

En cuanto a las habilidades según el género de los sujetos (tabla 5), no se aprecian diferencias significativas con $p=0,05$ que deban ser comentadas. No obstante, en general, entre las habilidades que presentan una menor frecuencia de observación se encuentran "Evaluar" (59\%) y "Predecir" (59\%) entre las mujeres y "Aplicar" (63\%) en hombres. Por el contrario, la habilidad "Inferir" se observa en hombres y mujeres por igual. Las frecuencias de observación se sintetizan en la tabla 5 . En definitiva, estos resulta- 
Tabla 4. Habilidades evaluadas mediante prueba escrita según carrera y frecuencia media de observación expresada como porcentaje de registros sobre el total de registros posibles para cada habilidad

\begin{tabular}{|c|c|c|c|}
\hline Habilidad & Matemática (\%) & Física (\%) & Química (\%) \\
\hline Aplicar & 86 & 75 & 50 \\
\hline Comparar & 65 & 100 & 67 \\
\hline Evaluar & 79 & 75 & 34 \\
\hline Inferir & 100 & 100 & 100 \\
\hline Plantear objetivos & 93 & 92 & 92 \\
\hline Predecir & 79 & 78 & 25 \\
\hline Uso de gráficos y tablas & 86 & 100 & 83 \\
\hline
\end{tabular}

Tabla 5. Habilidades evaluadas mediante prueba escrita según género y frecuencia media de observación expresada como porcentaje de registros sobre el total de registros posibles para cada habilidad

\begin{tabular}{|c|c|c|}
\hline Habilidad & Mujeres (\%) & Hombres (\%) \\
\hline Aplicar & 77 & 63 \\
\hline Comparar & 73 & 81 \\
\hline Evaluar & 59 & 69 \\
\hline Inferir & 100 & 100 \\
\hline Plantear Objetivos & 91 & 94 \\
\hline Predecir & 59 & 69 \\
\hline Uso de gráficos y tablas & 82 & 100 \\
\hline
\end{tabular}

dos están de acuerdo con lo que se reporta en cuanto a diferencia de género en las pruebas PISA para el caso chileno (OCDE, 2013).

\section{Conclusiones}

Al realizar la comparación de datos cualitativos y cuantitativos se establece una convergencia entre las observaciones de habilidades en la prueba escrita y el análisis de contenido de los registros audiovisuales. También se puede establecer la existencia de una recurrencia entre las estimaciones con diversos instrumentos y técnicas de observación y análisis.

Entre los principales resultados se encuentra que las habilidades testeadas se manifiestan sin distinción de género y sin diferencia significativa entre estudiantes de las carreras de pedagogía en física, en química y en matemática, hecho que destaca el potencial de la robótica para desarrollar habilidades de proceso científico en la formación inicial de profesores de ciencias y matemática. Este último punto debe ser entendido con carácter hipotético dado que en el contexto de la resolución de problemas de navegación con robot se observan de forma natural las habilidades reportadas. Es decir, si la intención es desarrollar este conjunto de habilidades, la robótica educativa nos proporciona un medio natural para conseguir este fin.

\section{Alcances y proyecciones}

Este es un estudio exploratorio, y aun cuando estos resultados no pueden ser generalizados, este caso representa una primera aproximación al estudio sistemático respecto del potencial de la robótica aplicada a la formación inicial de profesores de ciencias y matemática. En consecuencia, es necesa- 
rio implementar un diseño experimental con instrumentos confiables a fin de evaluar el potencial efectivo de la robótica educativa para desarrollar habilidades de proceso científico.

Por otra parte, entre las principales dificultades encontradas en el desarrollo se destaca la casi imposibilidad de dar cuenta de las habilidades de proceso científico reportadas en la literatura mediante un único instrumento para la recogida de datos. De ahí que se recurrió a diversos instrumentos, cada cual con sus factores a favor y en contra.
De todas formas debe enfatizarse en que es necesario desarrollar y probar más y mejores instrumentos junto a estrategias metodológicas adecuadas para la recogida de datos.

\section{Agradecimientos}

Los autores agradecen a la dirección de Investigación de la Universidad Metropolitana de Ciencias de la Educación por el apoyo al proyecto FIBE 14-12 del que se desprenden los resultados reportados.

\section{Referencias}

Abruscato, J. (2004). Teaching children science: Discovery methods for the elementary and middle grades (2 ed.). Boston: Pearson Education

Barker, B. y Ansorge, J. (2007). Robotics as Means o Increase Achievement Scores in an Informal Environment. Journal of Research on Technology in Education, 39 (3), 229-243

Barnes, D. J. (2002). Teaching introductory Java through Lego Mindstorms models. SIGCSE'O2 Proceedings of the 33rd SIGCSE technical symposium on Computer Science education, 147-151.

Beer, R. D., Chiel, H. J.y Drushel, R. F. (1999). Using autonomous robotics to teach science and engineering. Magazine Communications of the ACM, 42, 85-92.

Bybee, R. W. (1997). Towards an understanding of scientific literacy. Scientific Literacy, 37-68.

Cañedo Iglesias, C. (2008). Fundamentos teóricos para la implementación de la didáctica en el proceso enseñanza-aprendizaje. Recuperado el 22 de octubre de 2013 de: http://www.eumed.net/libros

Carbonaro, M., Rex, M. y Chambers J. (2004). Using LEGO robotics in a project-based learning environment. The Interactive Multimedia Electronic Journal of Computer-Enhanced Learning, 6 (1). Recuperado el 22 de octubre de 2013 de: http://imej.wfu.edu/articles/2004/1/02/index.asp\#7

Chambers, J. M. y Carbonaro, M. (2003). Designing, developing, and implementing a course on Lego robotics for technology teacher education. Journal of Technology and Teacher Education, 11, 209-241.

Chiappetta, D. y Koballa, T. (2006). Science instruction in the middle and secondary schools: Developing fundamental knowledge and skills for teaching (6 ed.). NJ: Pearson Prentice-Hall.

Else-Quest, N., Hyde, J. y Linn, M. (2010). Cross-national patterns of gender differences in mathematics: A metaanalysis. Psychological Bulletin, 136 (1), 103-127. 
Fagin, B. y Merkle, L. (2003). Measuring the effectiveness of robotics in teaching computer science. Proceedings of the 34rd SIGCSE technical symposium on computer science education. Recuperado el 22 de octubre de 2013 de: http://portal.acm.org/citation.cfm?id=611994\&coll=GUIDE\&dl=GUIDE\&CFID=11715560\&CFTOKEN=40703716

Fensham, P. J. (2000). Time to change drivers for scientific literacy. Canadian Journal of Science, Mathematics, and Technology Education, 2, 9-24.

Flake, J. (1990). An exploratory study of Lego logo. Journal of Computing in Childhood Education, 1, 15-22.

Flowers, T. R. y Gossett, K. A. (2002). Teaching problem solving, computing, and information technology with robots. Journal of Computing Sciences in Colleges, 17, 45-55.

Friedl, A. E. y Koontz, T. Y. (2005). Teaching Science to Children, An Inquiry Approach (6 ed.). NY: McGraw-Hill

García Y. y Reyes, D. (2012). Robótica Educativa y su potencial mediador en el desarrollo de las competencias asociadas a la alfabetización científica. Educación y Tecnología, 2, 42-55.

García, M. y McNeill, H. (2002). Learning how to develop software using the toy LEGO mindstorms. Proceedings of the 7 th annual conference on Innovation and technology in computer science education. ACM.

Gura, M. (2007). Student Robotic Classroom Robotics. En Case Stories of 21st Century Instruction for Millennial Students (pp. 11-31). Charlotte: Information Age Publishing.

Haapasalo, L.y Samuels, P. (2011). Responding to the challenges of instrumental orchestration through physical and virtual robotics. Computers y Education, 57, 1484-1492.

Klassner, F. (2002). A case study of LEGO Mindstorms'TM suitability for artificial intelligence and robotics courses at the college level. ACMSIGCSE Bulletin, 34, 8-12.

Klassner, F.y Anderson, S. (2003). LEGO mindstorms: Not just for K-12 anymore. IEEE Robotics and Automation Magazine, 10 (2), 12-18.

Kovalik, S.y Olsen, K. (2010). Kid's eye view of science: A conceptual, integrated approach to teaching science, K-6. USA:Corwin.

Kumar, A. (2004). Three years of using robots in an artificial intelligence course: lessons learned. Journal on Educational Resources in Computing, 4, 1-15.

Kuhn, D. (2000). Metacognitive Development. Current Directions in Psychological Science, 9, 177-181.

Laugksch, R. C. (2000). Scientific literacy. Science Education, 84 (1), 71-94.

Law, N. (2002). Scientific literacy: Charting the terrains of a multifaceted enterprise. Canadian Journal of Science, Mathematics, and Technology Education (2), 151-176.

Lundström, M. (2011). Decision-making in helth issues. Teenagers use of science and other discourses. Recuperado el 26 de octubre de 2013 de: http://dspace.mah.se/ 
ISSN 0123-1294 | Educ.Educ. Vol. 17. No. 2 | Mayo-Agosto de 2014 | pp. 271-285.

Universidad de La Sabana | Facultad de Educación

Lundström, M., Ekborg, M. y Ideland, M. (2012). To vaccinate or not to vaccinate: how teenagers justified their decision. Cultural Studies of Science Education, 7 (1), 193-221. doi: 10.1007/s11422-012-9384-4.

Martin, R., Sexton, C. y Franklin, T. (2009). Teaching science for all children: Inquiry methods for instructing understanding (4 ed.). Boston: Pearson Education.

Mauch, E. (2001). Using technological innovation to improve the problem solving skills of middle school students. The Clearing House, 75 (4), 211-213.

Mayer, V. J. y Kumano, Y. (2002). The Philosophy of Science and Global Science Literacy. En Mayer, V. J. (ed.). Global Science Literacy. Dordrecht: Kluwer Academic Publishers.

Miglino, O., Lund, H. H. y Cardaci, M. (1999). Robotics as an educational tool. Journal of Interactive Learning Research, 10, 25-48.

MINEDUC (2012). Bases curriculares 2012. Santiago de Chile: MINEDUC.

National Research Council NRC (1996). The National Science Standards. Recuperado el 25 de octubre de 2013 de: http://www.nap.edu/readingroom/books/nses.

Nourbakhsh, I. R., Crowley, K., Bhave, A., Hsium, T., Hammer, E.y Perez-Bergquist, A. (2005). The robotic autonomy mobile robotics course: Robot design, curriculum design and educational assesment. Autonomous Robots, $18(1), 103-127$.

Nourbakhsh, I. R. (2000). Robots and education in the classroom and in the museum: On the study of robots, and robots for study. Proceedings of workshop for personal robotics for education, institute of electrical and electronic engineers, international conference on robotics and automation. Salt Lake, Utah.

OCDE (1999). Measuring student knowledge and skills. A new framework for assessment. París: OCDE

OCDE (2004). Marcos Teóricos de PISA 2003: la medida de los conocimientos y destrezas en matemáticas, lectura, ciencias y resolución de problemas. Madrid: Ministerio de Educación y Ciencia, Instituto Nacional de Evaluación del Sistema Evaluativo (INECSE).

OCDE, (2007). PISA 2006. Science Competencies for Tomorrow's World, 1.

OCDE (2013). Resultados PISA 2012 Chile. Recuperado el 28 de octubre de 2013 de: http://www.agenciaeducacion. cl/wp-content/uploads/2013/O4/PISA-Programa-Internacional-de-Evaluaci\%C3\%B3n-de-Estudiantes.pdf

Papert, S. (1980). Mindstorms: Children, computers, and powerful ideas. New York: Basic Books, Inc.

Resnick, M. y Ocko, S. (1991). LEGO/logo: Learning through and about design. In Harel, I. y Papert, S. (eds.) Constructionism (pp. 141-150). Norwood, NJ: Ablex.

Ringwood, J. V., Monaghan, K. y Maloco, J. (2005). Teaching engineering through Lego Mindstorms. European Journal of Engineering Education, 30, 91-104. 
Robinson, M. (2005). Robotics-driven activities: Can they improve middle school science learning? Bulletin of Science, Technology \& Society, 25 (1), 73-84.

Rogers, C. y Portsmore, M. (2004). Bringing engineering to elementary school. Journal of STEM Education, 5 $(3,4), 17-28$.

Sargent, R., Resnick, M., Martin, F. y Silverman, B. (1996). Building and learning with programmable bricks. In Kafai, Y.y Resnick, M. (eds.). Constructionism in practice: Designing, thinking, and learning in a digital world (pp. 161-173). Mahwah, NJ: Lawrence Erlbaum.

Sendag, S.y Odabasi, F. (2009). Effects of an online problem based learning course on content knowledge acquisition and critical thinking skills. Computers y Education, 53, 132-141.

Shimbakuro, G. (1989). A class act: Junior high student lego and logo. The Computing Teacher, 16, 37-39.

Sullivan, F. R. (2008). Robotics and science literacy: Thinking skills, science process and system understanding. Journal of Research in Science Teaching, 45, 373-394.

van Eijck, M.y Roth, W. M. (2010). Theorizing scientific literacy in the wild. Educational Research Review, 5 (2), 184194.

Vázques-Alonso, Á., Acevedo-Díaz, J. A. y Manassero Mas, M. A. (2005). Más allá de la enseñanza de las ciencias para científicos: hacia una educación científica humanística. Revista Electrónica de Enseñanza de las Ciencias, 4 (2).

Wagner, S. P. (1998). Robotics and children: Science achievement and problem solving. Journal of Computing in Childhood Education, 9, 149-165.

Weinberg, J., Engel, G., Gu, K., Karacal, C., Smith, S., White, W., y Yu, X. (2001). A multidisciplinary model for using robotics in engineering education. In ASEE annual conference proceedings, 2001. Washington: American Society for Engineering Education.

Weinberg, J. B., White, W. W., Karacal, C., Engel, G. y Hu, A. P. (2005). A multidisciplinary teamwork in a robotics course. ACM SIGCSE Bulletin, 37, 446-450. 
